\title{
An Analysis of the Strengths and Weaknesses of Seoul Fashion Week with a Focus on Organisational Issues
}

\author{
Hye Eun $\operatorname{Kim}^{\dagger}$ \\ Fashion Womenswear, Royal College of Art; London, United Kingdom
}

\begin{abstract}
Seoul Fashion Week aims to be the world's fifth Fashion Week from the year after 2000. This has involved significant labour and investment, not all of which has been successful. This study analyses the strengths and weaknesses of SFW based on the London Fashion Week as a successful model. By identifying its obvious and less obvious characteristics, this analysis aims to diagnose SFW's current status in order to upgrade the event at an international level. Key factors are organisational body, designers, visitors, sponsors, schedule, venue, main events and supportive events. The following points have emerged from the research: the success of Seoul Fashion Week was based on the combination of separate collections, an appropriate fashion show venue, and the active role of new designers. The problems were: lack of an independent organisation, non-professional staff, lack of diverse visitors, exclusive governmental funding, late timing, excessive distance from the main European and American fashion markets, lack of sustainability of the main events, a limited variety of supportive events, the absence of online promotion and, last but not least, no distinctive features. The study concludes that Seoul Fashion Week has great potential with its fast growth and young designers. This should be complemented with an independent organisation and effective administration and strategy.
\end{abstract}

Key words : Seoul Fashion Week, organisation, buyers, governmental sponsorship, online promotion

\section{Introduction}

Seoul Fashion Week is a biannual fashion showcase event sponsored by the Seoul Metropolitan government. It aims to become the world's fifth leading Fashion Week, following London, Milan, New York and Paris (Seoul Design Foundation, 2015a). Although its 16-year-old history is short compared to others, its growth has been remarkable.

Several studies have been carried out on the subject of developing Seoul Fashion Week (hereafter, SFW): Analysis on the current status of Seoul Fashion Week and study on the methods of its mid- and long-term development (Kim, 2012), Research on "Korean" collection as high added value fashion industry -by comparison of status and characteristics of domestic and international collections (Joo, 2009), The development strategies of SFAA, Seoul collection (Kim, 2008a), The development strategies of the Seoul fashion industry (Kim, 2008b) and An exploratory study for the market of Seoul collection - from the collection participants' perspective (Han \& Lee, 2008). A number of press articles (Ham, 2011; Kim, 2015; Lee, 2013; Lee, 2014), report the strengths and weaknesses of each collection, particularly in organisational terms. Organisational issues are crucial for assessing the present as well as planning future directions. It is also important

$\uparrow$ Corresponding author; Hye Eun Kim

Tel. +82-2-2248-5040, Fax. +82-2-2213-9355

E-mail: hyk0010@hotmail.com to look into the success and problems in order to construct a correct and realistic development plan. However, no detailed academic studies about SFW organisational issues were made available after the year 2012, when separate collections were combined into one, despite the great quantity of news and magazine articles on this topic. The aim of this paper is to define the current situation of SFW, particularly after 2012. To do this, it is helpful to look at the key factors of some successful events of this kind. London Fashion Week (LFW) can be a good model as an independent, yet powerful, organisation.

The scope of this research focuses on the changes in SFW after 2012, particularly in terms of organisational issues. The issues of designers, prices and products are as important as organisational ones in order to produce an accurate diagnosis, as these, however, would be too wide to be considered in one paper, this study only focuses on organisational points. The research method is based on a review of the relevant literature, including online resources and news articles. With its efficient organisational body, the British Fashion Council (BFC) and its successful strategy (guests, sponsors, events etc.), LFW will be used as a standard against which to assess the current situation of SFW. It seems to be best to investigate both websites (i.e., LFW's and BFC's) because buyers and press alike regard Fashion Week websites as the best way to gain information about designers, schedules and events. This is confirmed by interviews (from other research projects) with buyers (7), press (21), stylists (2), photographers (1) and makeup artists (1) 
during 2015 SS LFW, 12-16 September 2014. All of them mention the homepage as their best information source. Then, by the standards from websites, SFW is investigated through journals, news articles and related websites, SFW, CFDK (Council of Fashion Designers of Korea), designers' websites and reports by governmental organisations (Seoul Fashion Centre, Seoul Institute, Seoul Metropolitan Government and Seoul Design Foundation). This study aims to illustrate unnoticed success along with more obvious achievements, in order to devise specific strategies for SFW to become the world's fifth Fashion Week and launch Korean fashion internationally.

\section{The key factors to consider with a focus on organisation}

\subsection{The key factors of Fashion Week in terms of funding and organisation}

Fashion Week is a preview chance of the latest trend and tradebased events (Kim, 2015). The world's four biggest Fashion Weeks are held in London, Milan, New York and Paris. These are followed by similar events in Berlin, Tokyo, Los Angeles, Istanbul, and other cities. New York Fashion Week is run by IMG Fashion, the world's leading producer, marketer and partner to the international fashion community. Although New York Fashion Week was established by the Council of Fashion Designers of America, it was handed over to IMG in 2001. New York Fashion Week was funded by Mercedes-Benz from 2007 until January 2015. Following this successful sponsorship, Mercedes-Benz has funded other Fashion Weeks, such as Australia, Berlin, China, Istanbul, New York and Tokyo (Mercedes-Benz Fashion Week Homepage, 2015). Paris is run by the French Fashion Federation and funded by private corporations such as Mercedes-Benz and Maybelline, New York. Milan Fashion Week is run by the National Chamber for Italian Fashion, a non-profit organisation, and supported by the City of Milan and other private companies although it is not clear whether this governmental support is financial or institutional help.

London Fashion Week is run by BFC and its main funder is the
City of London, followed by the London Development Agency, Department for Business, Innovation and Skills, and the EU's European Regional Development Fund. However, these sponsorships are for the Paris Fashion Week showroom fee. Normally most contracts are made during Paris Fashion Week, at the end of the Big 4 Fashion Weeks. Buyers confirm their contracts after seeing the major Fashion Weeks. So many LFW designers are represented in Paris showrooms during Paris Fashion Week. The City of London contributes 800,000 British pounds ( 1,440,000,000, 1,800= $£ 1$ ). This is just $8 \%$ of the total expenses (Seoul Institute, 2014). The rest is covered by business sponsors (products, services or money), by profits from the fashion weekend, a consignment fee, and designers' fees.

Tokyo Fashion Week is run by the Japan Fashion Week Organization and used to be funded by Tokyo's city government until 2010. Now two thirds of the expenses ( 4,000,000,000) are covered by sponsorship from various companies. Berlin Fashion Week is run by Berlin Partner and supported by the title sponsor, Mercedes-Benz. Singapore Fashion Week (Audi Fashion Festival) is run by Asia Fashion Exchange, and its biggest sponsor (half of their budget) is Audi. The rest is from governmental organisations such as the Small and Medium Business Administration and STB (Singapore Tourism Board). SFW is currently hosted by Seoul Metropolitan Government and run by Seoul Design Foundation. Seoul City is almost the sole funder of Fashion Week (only 5\% is from other sponsorships) (ibid.).

Fashion Week sponsorship sources can be classified as private corporations and governmental funding. Independent sponsorships are from private corporations (New York, Berlin and Tokyo) and are divided into profit- and non-profit making. There is a mix of governmental and company funding (Singapore), and governmental funding (Seoul). Fig. 1 shows the differences in funding each Fashion Week.

Among these, LFW is a suitable model for SFW to follow. Singapore Fashion Week tends to host well-known designers' garments shown at an earlier major Fashion Week, rather than run its own fashion show (Seoul Metropolitan Government Fashion Advi-

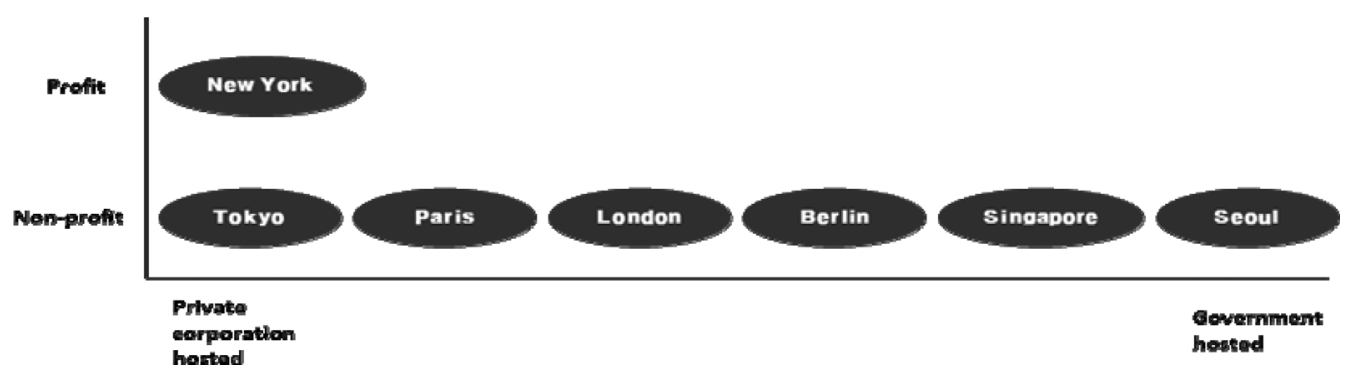

Fig. 1. Funding and profit- or non-profit making of each Fashion Week. (modified from Seoul Institute, 2014) 
Table 1. Key factors from LFW and SFW Homepage (2016 SS)

(Retrieved 28 August 2015)

\begin{tabular}{|c|c|c|c|c|c|c|c|c|}
\hline & $\begin{array}{l}\text { Organisational } \\
\text { body }\end{array}$ & $\begin{array}{l}\text { Participants } \\
\text { (Designers) }\end{array}$ & $\begin{array}{l}\text { Visitors } \\
\text { (Guests) }\end{array}$ & Sponsor (funder) & Schedule & Venue & Events & Supportive events \\
\hline London & $\begin{array}{l}\text { British } \\
\text { Fashion } \\
\text { Council- have } \\
\text { separate } \\
\text { homepage } \\
\text { about } \\
\text { business, } \\
\text { event etc. }\end{array}$ & $\begin{array}{l}\text { Contact details } \\
\text { (sales, press), } \\
\text { stock list, } \\
\text { showroom, } \\
\text { company } \\
\text { details, } \\
\text { social media, } \\
\text { look book }\end{array}$ & $\begin{array}{l}\text { Press (including } \\
\text { bloggers) and } \\
\text { buyers, press, } \\
\text { buyers } \\
\text { registration, } \\
\text { press portal, } \\
\text { galleries for } \\
\text { press }\end{array}$ & $\begin{array}{c}\text { Principal } \\
\text { sponsor, } \\
\text { other sponsors, } \\
\text { suppliers, } \\
\text { funders (EU, } \\
\text { Mayor of } \\
\text { London, UK } \\
\text { Trade \& } \\
\text { Investment) }\end{array}$ & $\begin{array}{l}18-22 \\
\text { Sep. }\end{array}$ & $\begin{array}{c}\text { The Brewer Street } \\
\text { Car Park ( }\left(^{\text {st }} \text { time }\right) \\
(2010-15: \\
\text { Somerset House }\end{array}$ & $\begin{array}{l}\text { Catwalk } \\
\text { show, } \\
\text { showroom, } \\
\text { digital } \\
\text { show, } \\
\text { film } \\
\text { screening }\end{array}$ & $\begin{array}{c}\text { Pop-Up showroom, Unfold } \\
\text { (Irish Designers showcase), } \\
\text { photographic } \\
\text { exhibition, Lovebullets } \\
\text { ART exhibition, after } \\
\text { Party, Louis Vuitton Series } \\
3 \text { exhibition opening } \\
\text { gala, Belstaff event etc. }\end{array}$ \\
\hline Seoul & $\begin{array}{l}\text { Hosted by } \\
\text { Seoul } \\
\text { Metropolitan } \\
\text { Government } \\
\text { Run by Seoul } \\
\text { Design } \\
\text { Foundation }\end{array}$ & $\begin{array}{c}\text { Contact, } \\
\text { designer } \\
\text { profile, brand } \\
\text { concept, season } \\
\text { concept, } \\
\text { collection } \\
\text { photos -no link } \\
\text { to } \\
\text { homepage \& } \\
\text { social media }\end{array}$ & $\begin{array}{l}\text { Press, buyers } \\
\text { registration }\end{array}$ & N/A & $\begin{array}{l}16-21 \\
\text { Oct. }\end{array}$ & $\begin{array}{c}\text { Dongdaemun } \\
\text { Design Plaza } \\
\text { (from } 2014 \mathrm{AW} \text { ) }\end{array}$ & $\begin{array}{c}\text { Seoul } \\
\text { Collection } \\
\text { Generation } \\
\text { Next } \\
\text { Off show }\end{array}$ & Give Donation \\
\hline
\end{tabular}

sory Team, 2009). SFW has not developed enough yet to be supported by a main sponsor such as Mercedes Benz. In fact, a big multinational company as a main sponsor using its own name would seem clash with SFW's intention to encourage the domestic industry. Further, relying on just one company for sponsorship makes it difficult to replace it when the contract ends. Thus, a better option for SFW seems to be to find a number of small, separate sponsors in order to decrease dependency on the government. Paris Fashion Week has various collections and fairs, including Prêt a Porter, Haute Couture, Who's Next, among others. However, because of its scale and complexity, it is not the best model for SFW to follow. Although Milan Fashion Week is funded by the City of Milan and LFW is funded by London's city government, both are run by non-profit organisations, recruit sponsorships and run businesses in order to fund their Fashion Weeks and associated events. However, LFW is famous as a gateway for new designers and its program is aimed at nurturing young designers. Of all designers participating in $2015 \mathrm{AW}, 44.87 \%$ have run their own brands for less than 5 years 35 out of 78, including Generation Next and Seoul Collection (the details are in tables 4 and 2.2.1. Designers). Given the difficulty for new designers to sustain their labels, BFC, an organiser of LFW, can be taken as an ideal sample model to diagnose the strengths and weaknesses of SFW so as to set up a future plan. In Table 1, information from the LFW website is compared with the corresponding aspects of SFW.

\subsection{The key factors of SFW}

Drawn from LFW and BFC homepages, these factors encom- pass: organisational body, designers, visitors, sponsor, date, venue, events and supportive events. Table 2 shows the changes in SFW from 2012 AW.

\subsubsection{Organisational body}

The first fashion show in Korea was by Nora Noh on 29th November 1959 at the Bando Hotel (Noh, 2007). The first regular group fashion show started from SFAA (Seoul Fashion Association) 1991 SS (November, 1990). In the 1990s there were a number of similar well-established fashion shows showcasing events such as the KDFA, SFAA and NWS Seoul Collections. SFAA, established in 1989, is led by designers from the Kookje Design Institute or the Choongang Design Contest. Since then, it has been holding two fashion shows every year. KFDA (Korea Fashion Designers Association), established in 1961, is based on boutique designers near Myoungdong and NWS (New Wave in Seoul), established in 1992 by new and young designers, when SFAA and KFDA were already established (Park, 2000). Because of the different interests and goals, collections were held separately, despite Seoul City's combining efforts. In 1998, the Ministry of Trade, Industry \& Energy, together with Seoul Metropolitan Government, launched Seoul Collection as a event of ASEM which combined KFDA, NWS and other independent designers. In October 2000, Seoul Collection launched its first fashion shows. After that, there have been two fashion collections in Korea: the Seoul Collection and the SFAA Collection. Shows' schedules, ticket sales and PR are organised separately. This is wasteful of labour, money and PR, and weakens the collections in terms of 
Table 2. Changes from $2012 \mathrm{AW}$

\begin{tabular}{|c|c|c|c|c|c|c|c|c|}
\hline Season & $\begin{array}{l}\text { Organisation } \\
\text { al body }\end{array}$ & $\begin{array}{c}\text { Designers } \\
\text { : catwalk show }\end{array}$ & $\begin{array}{l}\text { Visitors } \\
\text { (Guests) }\end{array}$ & $\begin{array}{l}\text { Sponsor-funder } \\
\text { : Seoul City }\end{array}$ & $\begin{array}{c}\text { Date } \\
\text { (Schedule) }\end{array}$ & Venue & Events & Supportive events \\
\hline 2012 AW & SDF & $\begin{array}{l}67 \\
\text { (SC: } 36, \text { FTO: } \\
19, \\
\text { GN: } 12)\end{array}$ & & \multirow{2}{*}{$\begin{array}{c}2012 \\
-3.875 \text { billion } \\
(3,875,000,000)\end{array}$} & $\begin{array}{l}\text { 2-7 April } \\
\text { (6 days) }\end{array}$ & $\begin{array}{l}\text { Olympic } \\
\text { Park }\end{array}$ & $\begin{array}{l}\text { SFF- } \\
200\end{array}$ & GIVE Donation \\
\hline $2013 \mathrm{SS}$ & SDF & $\begin{array}{c}56 \\
\text { (SC :41, GN: } 12 \text {, } \\
\text { OS: } 3 \text { ) }\end{array}$ & & & $\begin{array}{c}22-28 \text { Oct. } \\
\text { (7 days) }\end{array}$ & $\begin{array}{l}\text { War } \\
\text { Memorial } \\
\text { Museum, } \\
\text { Xi Gallery }\end{array}$ & $\begin{array}{l}\text { SFF- } \\
200\end{array}$ & GIVE Donation \\
\hline 2013 AW & SDF & $\begin{array}{c}70 \\
\text { (SC: } 50, \text { GN: } 11, \\
\text { OS: } 9 \text { ) }\end{array}$ & & \multirow[b]{2}{*}{$\begin{array}{c}2013 \\
-3.1 \text { billion } \\
(3,100,000,000)\end{array}$} & $\begin{array}{l}\text { 25-30 Mar. } \\
\text { (6 days) }\end{array}$ & $\begin{array}{l}\text { IFC Mall, } \\
\text { Blue } \\
\text { Square }\end{array}$ & SFF-? & GIVE Donation \\
\hline $2014 \mathrm{SS}$ & $\begin{array}{l}\text { SDF (27), } \\
\text { CFDK (25) } \\
\text { (run each } \\
\text { shows } \\
\text { separately) }\end{array}$ & $\begin{array}{c}70 \\
\text { (SC: } 52, \mathrm{GN}: 18)\end{array}$ & $\begin{array}{c}62,655 \\
\text { (IB-124, } \\
\text { IP-53) }\end{array}$ & & $\begin{array}{c} \\
18-23 \text { Oct. } \\
(6 \text { days })\end{array}$ & $\begin{array}{c}\text { Hangang } \\
\text { Park, IFC } \\
\text { Mall }\end{array}$ & SFF-? & $\begin{array}{l}\text { Asian Fashion Forum, Art zone, } \\
\text { Camping zone, K-style Concert, } \\
\text { Pop-up stores, 800g fashion } \\
\text { donation, Galleria West pop up } \\
\text { store by Singapore designers at } \\
\text { SFW, Fashion filming (Nora Noh) }\end{array}$ \\
\hline 2014 AW & $\begin{array}{l}\text { SDF, } \\
\text { CFDK }\end{array}$ & $\begin{array}{c}86 \\
\text { (SC: 60, GN: } 17 \text {, } \\
\text { OS: 9) }\end{array}$ & & \multirow{2}{*}{$\begin{array}{c}2014 \\
-3 \text { billion } \\
(3,003,034)\end{array}$} & $\begin{array}{l}\text { 21-26 Mar. } \\
\text { (6 days) }\end{array}$ & DDP & SFF-48 & $\begin{array}{l}\text { Fashion Collaboration-EXO+ GN, } \\
\text { GIVE Donation } \\
\text { (etc. Singapore Fashion Week } \\
\text { Trade Show-Blue Print attending) }\end{array}$ \\
\hline $2015 \mathrm{SS}$ & $\begin{array}{l}\text { SDF, } \\
\text { CFDK }\end{array}$ & $\begin{array}{c}86 \\
\text { (SC: } 60, \mathrm{GN}: 17 \text {, } \\
\text { OS: 9) }\end{array}$ & $\begin{array}{c}55,558 \\
\text { (IB-212, IP-308, } \\
\text { B-114, P-786) }\end{array}$ & & $\begin{array}{l}\text { 17-22 Oct. } \\
(6 \text { days })\end{array}$ & DDP & $\begin{array}{r}\text { SFF-33 } \\
(\text { PT-5) }\end{array}$ & GIVE Donation \\
\hline 2015 AW & $\begin{array}{c}\text { SDF } \\
\text { (Sponsor- } \\
\text { CFDK) }\end{array}$ & $\begin{array}{c}83 \\
\text { (SC: } 57, \text { GN: } 21, \\
\text { OS: } 5 \text { ) }\end{array}$ & & $\begin{array}{l}2015 \text { (including } \\
2016 \mathrm{SS} \text { ) } \\
-2.7 \text { billion } \\
(2,704,954)\end{array}$ & $\begin{array}{l}\text { 20-25 Mar. } \\
\text { (6 days) }\end{array}$ & DDP & No & GIVE Donation \\
\hline
\end{tabular}

* From the year 2012, all financial support has been from Seoul Metropolitan City Government.

* $\mathrm{SC}=$ Seoul Collection, $\mathrm{GN}=$ Generation Next, $\mathrm{FTO}=$ Fashion Take Off, OS= Off Show, SFF $=$ Seoul Fashion Fair

* $\mathrm{IB}=$ International Buyer, $\mathrm{IP}=$ International Press, $\mathrm{B}=$ Buyer, $\mathrm{P}=$ Press

attracting international attention to one city. Finally, after significant efforts towards unification, the year 2003 saw the first combined SS collection. However, this did not last beyond that year because of distribution problems in scheduling shows, fee administration, funding, tickets, etc. Until 2012 there were separation and unification in succession. Finally, one collection was held in 2012. It was hosted by the Seoul Metropolitan Government and co-run by Seoul Design Foundation and CFDK (Council of Fashion Designers of Korea). CFDK was established in 2012 by combining separate designer groups, in order to "create a truly unified fashion designer association that will bring every fashion designer organizations and individual designers together" (Council of Fashion Designers of Korea, 2015). Since then, CFDK has worked together with, or against, SDF for SFW. Table 3 shows the chronological order of fashion collections in Korea and Seoul Fashion Week's organisational bodies.

\subsubsection{Designers}

There are various types of designers showing exhibiting at the
Fashion Week (Table 4). Almost half of them have run their businesses for less than five years. It is hard to survive for young brands because of financial problems, weak brand identity, inability to keep regular customers, etc. This is why a nurturing program is needed. SFW has Generation Next, which supplies venues on a smaller scale than is needed for Seoul Collection. This is a chance for new designers to operate on smaller budgets, participate in shows and contact buyers, press, and even the public.

\subsubsection{Guests}

Unlike other Fashion Weeks, SFW is open to any ticket holder. Therefore, the guests include press, buyers, VIPs, celebrities, students, etc. Conversely, the four major Fashion Weeks are tradeonly events, only open to press, buyers and VIPs. In 2015 SS, the number of visitors to SFW was 55,558, whilst the visitors to LFW were 5,000, that is, almost ten times less. Moreover, contract amounts were significantly different (See Table 5).

2.2.4. Sponsorship 
Table 3. Collections and organising bodies in chronological order

\begin{tabular}{|c|c|c|c|}
\hline & Number of collections & Organising bodies & Funder of collection \\
\hline $1990 \mathrm{~s}$ & Three (KFDA, SFAA, NWS) & KFDA, SFAA, NWS & different funders \\
\hline $\begin{array}{l}2001 \text { SS } \\
(\text { Oct, 2000) }\end{array}$ & $\begin{array}{l}\text { Two: Seoul Collection (KFDA, NWS) } \\
\text { SFAA Collection }\end{array}$ & \multirow{6}{*}{ Korea Fashion Association, SFAA } & \multirow{6}{*}{$\begin{array}{l}\text { Seoul Metropolitan Government, } \\
\text { Ministry of Trade \& Industry }\end{array}$} \\
\hline $2003 \mathrm{SS}$ & One: Seoul Collection & & \\
\hline 2003 AW & Two: Seoul Collection, SFAA Collection & & \\
\hline $2004 \mathrm{SS}$ & One in two locations & & \\
\hline 2007 AW & $\begin{array}{c}\text { Separate: Seoul Collection, SFAA } \\
\text { Collection }\end{array}$ & & \\
\hline $2009 \mathrm{SS}$ & Two: Seoul Collection, SFAA Collection & & \\
\hline 2012 SS & Two: Seoul Collection, SFAA Collection & $\begin{array}{l}\text { Seoul Fashion Centre (in Seoul Business } \\
\text { Agency), SFAA }\end{array}$ & $\begin{array}{l}\text { Seoul Metropolitan Government, } \\
\text { Ministry of Knowledge Economy }\end{array}$ \\
\hline 2012 AW & One- Seoul Collection & Seoul Design Foundation & \multirow{3}{*}{$\begin{array}{l}\text { All financial support is from Seoul } \\
\text { Metropolitan Government }\end{array}$} \\
\hline 2014 SS-2015 SS & Seoul Collection & $\begin{array}{c}\text { Seoul Design Foundation, CFDK } \\
\text { : MOU between two } \\
\text { (Run by Seoul (27), CFDK (25) in } 2014 \text { SS) }\end{array}$ & \\
\hline 2015 AW & Seoul Collection & Seoul Design Foundation (CFDK: Sponsor) & \\
\hline
\end{tabular}

* SFC was closed in December 2011 and all responsibilities were handed over to SDF. (Seoul Institute, 2014)

Seoul Metropolitan Government has been the sole funder from 2012 AW, contributing 3.1 billions in 2013 , that is, $29.9 \%$ of the government's fashion budget made available in 2013 (Seoul Institute, 2014). This figure keeps decreasing: 4.9 billion in 2008, 3.1 billion in 2013 and 2.7 billion in 2015. As mentioned earlier, sponsorship from companies has only covered $5 \%$ of the total expenses in SFW. However, LFW distinguishes between main sponsor, other sponsors, suppliers, and funders contracted seasonally by BFC.

\subsubsection{Time}

SFW is held in March and October. Table 6 is a 15 AW schedule of the world's Fashion Weeks. Buyers and press travel from New York to London, Milan and Paris without break. Buyers interested in new Asian brands keep their leftover budget for Asia, mostly Tokyo. However, most of the buying is done by the end of the Big 4.

\subsubsection{Place}

Collection venues have changed much through time. Some were more proper for fairs or exhibitions than fashion shows and have been criticised for not representing Korean tradition, the good features of Seoul, the current development of the Korean fashion industry, as well as for their inadequate accessibility by transport (Han \& Lee, 2008; Kim, 2012). From 2000 to 2004 AW, SFW was held at Coex, then it was moved to SETEC (near Hagnyeoul Station) from 2005 SS to 2012 SS. In 2012 AW, it took place at the Olympic Park at Jamsil, and then moved to the War Memorial Museum and Riverside Park, IFC Mall. Finally, in 2014 AW,
DDP (Dongdaemoon Design Plaza) was open and SFW was held there.

\subsubsection{Event}

SFW included Seoul Collection and Generation Next in 2015 AW, and will do so again in 2016 SS. Seoul Collection is the biggest business showcase for established designers. Generation Next represents 1-5-year-old designer brands and is a gateway for the new generation. There is Off Show as well, taking place outside DDM.

Until 2015 SS there had been Seoul Fashion Fair for trade shows including menswear, womenswear and accessories (50 or more companies), with the chance of a presentation show. It also featured GIVE Donation, a donation market open to public (no entrance fee) showcasing donated designer products.

\section{Strengths}

Despite its relatively short history, the growth of SFW has been remarkable. Its size is huge (around 55,000 visitors) and the number of shows is as large as that of 31-year-old LFW.

\subsection{One organisation}

SFW had suffered from the fragmentation of its organisational bodies, the main drawback to its development (Joo, 2009; Kim, 2012; Seoul Institute, 2014). Finally, in 2012 AW, one combined SFW was held and solely run by Seoul Design Foundation, funded by Seoul Metropolitan Government since 2008, 13 years after the 
Table 4. The running years of participating brands in 2015 AW SFW Seoul Collection

\begin{tabular}{|c|c|c|c|c|c|c|c|c|c|c|c|c|}
\hline 20 , Mar & Brand & SONGZIO & $\begin{array}{l}\text { Ordinary } \\
\text { People }\end{array}$ & $\begin{array}{l}\text { MUNSOO } \\
\text { KWON }\end{array}$ & $\begin{array}{l}\text { VanHart di } \\
\text { Albarzar }\end{array}$ & $\begin{array}{l}\text { HEICH ES } \\
\text { HEICH }\end{array}$ & beyond closet & Jehee Sheen $S$ & SLING STONE & & & \\
\hline & Director & Zio Song & $\begin{array}{l}\text { Hyuncheol } \\
\text { Chang }\end{array}$ & $\begin{array}{c}\text { Munsoo } \\
\text { Kwon }\end{array}$ & $\begin{array}{l}\text { Dooyoung } \\
\text { Chung }\end{array}$ & Sanghyuk Han & Taeyong Ko & Jehee Sheen & Jongcheol Park & & & \\
\hline & Year & 1992 & 2013 & 2011 & 2011 & 2014 & 2008 & 2009 & 2006 & & & \\
\hline \multirow[t]{3}{*}{ 21, Mar } & Brand & THE CENTAUR & kimseoryong & $\begin{array}{l}\text { ANS AN } \\
\text { YOONJUNG }\end{array}$ & CARUSO & La doii & $\begin{array}{l}\text { RESURRECTI } \\
\text { ON }\end{array}$ & the studio $\mathrm{K}$ & $\mathrm{J} \mathrm{KOO}$ & SONO DRS & $\begin{array}{c}\text { SUPERCOMM } \\
\text { A } \\
\text { B }\end{array}$ & \\
\hline & Director & Ranji Ye & Seoryong Kim & Yoonjung An & $\begin{array}{l}\text { Kwanhyo } \\
\text { Chang }\end{array}$ & Doii, Lee & Jooyoung Lee & Hyejin Hong & $\begin{array}{l}\text { Jinwoo Choi, } \\
\text { Yeonjoo Koo }\end{array}$ & Deresa Choi & Bonnie Lee & \\
\hline & Year & 2008 & 1998 & 1975 & 1987 & 2008 & 2004 & 2006 & 2011 & 2012 & 2015 & \\
\hline \multirow[t]{3}{*}{ 22, Mar } & Brand & $\begin{array}{c}\text { POST } \\
\text { DECEMBER }\end{array}$ & $\begin{array}{l}\text { Kwakhyunjoo } \\
\text { collection }\end{array}$ & $\begin{array}{l}\text { PAUL\& } \\
\text { ALICE }\end{array}$ & $\begin{array}{l}\text { LE QUEEN } \\
\text { couture }\end{array}$ & sumisumi & PartspARTs & NOHKE & CHOIBOKO & $\begin{array}{l}\text { SOULPOT } \\
\text { STUDIO }\end{array}$ & how and what & \\
\hline & Director & Sohyun Park I & Hyunjoo Kwak & Hyosoon Joo & $\begin{array}{l}\text { Youseok } \\
\text { Moyung }\end{array}$ & Sumi Jeong & Seonok Im & Misun Chung & Bokho Choi & Soojin Kim & Byoungkyu Park & \\
\hline & Year & 2009 & 2003 & 2010 & $2007 / 8$ & 2012 & 2011 & 2011 & 1973 & 2009 & 2003 & \\
\hline \multirow[t]{3}{*}{ 23, Mar } & Brand & $\begin{array}{c}\text { FASHION } \\
\text { STORY JUNG } \\
\text { HUN JONG }\end{array}$ & $\begin{array}{c}\text { LIE } \\
\text { SANGBONG }\end{array}$ & $\begin{array}{l}\text { KAAL } \\
\text { E. SUKTAE }\end{array}$ & $\begin{array}{c}\text { Push- } \\
\text { BUTTON }\end{array}$ & $\begin{array}{l}\text { KUMANN } \\
\text { YOO HYE JIN }\end{array}$ & $\begin{array}{c}\text { MISS GEE } \\
\text { COLLECTION }\end{array}$ & BIG PARK & $\begin{array}{l}\text { Steve J \& } \\
\text { Yoni P }\end{array}$ & ARCHE & METROCITY & $\begin{array}{l}\text { KIM CHUL } \\
\text { UNG MODE }\end{array}$ \\
\hline & Director & Hunjong Jung & Sangbong Lie & Suktae Lee & $\begin{array}{l}\text { Seongkeon } \\
\text { Park }\end{array}$ & Hyejin Yoo & Choonhee Gee & Yonsoo Park & $\begin{array}{l}\text { Hykseo Jung, } \\
\text { Seunyeon Pae }\end{array}$ & Choonho Yoon & & Chulwoong Kim \\
\hline & Year & $2008 ?$ & 1985 & 1997 & 2003 & 2012(1979) & 1979 & 2011(1980) & 2006 & 2013 & 1992 & $1979 / 80$ \\
\hline \multirow[t]{3}{*}{ 24, Mar } & Brand & ENZUVAN & $\begin{array}{c}\text { DEMOO } \\
\text { PARKCHOON } \\
\text { MOO }\end{array}$ & CRES E DIM & Jain Song & $\mathrm{S}=\mathrm{YZ}$ & JARRET & KYE & BAKANGCHI & & & \\
\hline & Director & Eunzu Hong & Choomoo Park I & Hongbum Kim & Jain Song & Yoojin Song & Jiyeon Lee & Hanhee Kye & Hangchi Park & & & \\
\hline & Year & 2000 & 1988 & 2009 & 2004 & 2013 & 2009 & 2012 & 1973 & & & \\
\hline \multirow[t]{3}{*}{ 25, Mar } & Brand & Monte Milano & SUUWU & $\begin{array}{l}\text { yang's by } \\
\text { HEE DEUK }\end{array}$ & CANEZOU & kiok & $\begin{array}{c}\text { JO MYUNG } \\
\text { RYE }\end{array}$ & $\begin{array}{l}\text { MAG \& } \\
\text { LOGAN }\end{array}$ & $\begin{array}{c}\text { LOW } \\
\text { CLASSIC }\end{array}$ & ANDY \& DEBB & ultimo & \\
\hline & Director & Seohee Oh & Suuwu Park & Heedeuk Yang & Bomin Kim & Kiok Kang & Myungrye Jo & $\begin{array}{l}\text { Logan, Sunhye } \\
\text { Chung }\end{array}$ & Mungsin Lee & $\begin{array}{l}\text { Seokwon Kim, } \\
\text { Wonjung Yoon }\end{array}$ & Dongsoon Kim & \\
\hline & Year & $2003 ?$ & $2012 ?$ & $2007(2000)$ & 2006 & 1989 & 1977 & 2006 & $2009 / 10$ & 1999 & 1985 & \\
\hline
\end{tabular}

* As Generation Next (21 designers) consists of designers who have been in business for under 5 years, this table does not include their launching year. Because of their small size, it is hard to find information of some designers.

* Renamed brands, such as KUMANN YOO HYE JIN or BIG PARK are included in the original brands' launching year rather than their rebranding year. Despite reforming and rebranding, these companies have kept the same organisation and funding power. 
Table 5. Number of visitors and amount of contracts at 2015 SS SFW and LFW

\begin{tabular}{|c|c|c|}
\hline & 2015 SS SFW & 2015 SS LFW \\
\hline Date & 17 (Fri)-22 (Wed). Oct. 2014 (6days) & 12(Fri)-16 (Tue). Sep. 2014 (5 days) \\
\hline Participating Designers & $\begin{array}{l}\text { Catwalk show } 86 \\
\text { Fair } 33\end{array}$ & $\begin{array}{l}\text { Catwalk show } 82 \\
\text { Showroom } 170\end{array}$ \\
\hline Number of Visitors & $\begin{array}{l}55,558 \\
\text { (including designer's guests, students, etc.) } \\
\text { International Buyers: } 212 \text {, } \\
\text { Domestic Buyers: } 114, \\
\text { International Press: } 308, \\
\text { Domestic Press: } 786\end{array}$ & $\begin{array}{l}5,000 \\
\text { (buyers, journalists, bloggers, broadcast crews, } \\
\text { photographers)* }\end{array}$ \\
\hline Total value of Contracts & $\$ 2,000,000$ & $\$ 155,000,000$ (£ 100 million $)$ \\
\hline
\end{tabular}

* The numbers of buyers, press and others are not shown separately.

Table 6. Fashion Week Schedule

\section{February}

\begin{tabular}{|c|c|c|c|c|c|c|}
\hline Sun & Mon & Tue & Wed & Thu & Fri & Sat \\
\hline B & 9 & 10 & 11 & 12 & 13 & 14 \\
\hline & & & & \multicolumn{3}{|c|}{ New York } \\
\hline 15 & 16 & 17 & 18 & 19 & 30 & 21 \\
\hline \multicolumn{5}{|c|}{ New York } & \multicolumn{2}{|c|}{ London } \\
\hline 22 & 23 & 24 & 25 & 26 & 27 & 28 \\
\hline \multicolumn{3}{|c|}{ London } & \multicolumn{4}{|c|}{ Milan } \\
\hline & & & & & \multicolumn{2}{|c|}{ March } \\
\hline Sun & Mon & Tue & Wed & Thu & Fri & Sat \\
\hline 1 & 2 & 3 & 4 & 5 & 6 & 7 \\
\hline \multicolumn{2}{|c|}{ Milan } & & \multicolumn{4}{|c|}{ Paris } \\
\hline \multirow[t]{2}{*}{8} & 9 & 10 & 11 & 12 & 13 & 14 \\
\hline & & & & & \\
\hline 15 & 16 & 17 & 18 & 19 & 20 & 21 \\
\hline & & \multicolumn{5}{|c|}{ Tokyo } \\
\hline & & & & & \multicolumn{2}{|c|}{ Seoul } \\
\hline 22 & 23 & 24 & 25 & 26 & 27 & 28 \\
\hline \multicolumn{7}{|c|}{ Tokyo } \\
\hline \multicolumn{7}{|c|}{ Seoul } \\
\hline & & & \multicolumn{4}{|c|}{ China } \\
\hline 29 & 30 & 31 & & & & \\
\hline \multicolumn{7}{|c|}{ China } \\
\hline
\end{tabular}

launch of Seoul Collection. This consolidation can help upgrade SFW to a world-class Fashion Week.

\subsection{Venue}

Another issue has been the venue. Previous options, such as SETEC, on the outskirts of Seoul, were more suitable for trade fairs. Visiting individual brand showrooms was inconvenient especially for buyers, who usually prefer this way of gleaning information about companies. SETEC had been built to host trade shows, not fashion events (Han \& Lee, 2008), and cannot represent the cultural heritage of Korea (Kim, 2012). After some experi- menting (Olympic Park, War Memorial Museum, IFC, Yeouido Park), SFW was finally held at DDP. DDP represents the cultural advancement of Korea, particularly in fashion and design (Dongdaemoon Design Plaza, 2015). It is situated near Dongdeaemoon, a fashion market and industry hub in Seoul, centrally located and well served by public transport, with easy access to designers' showrooms and historic sites such as Gyeongbokgung Palace and Deoksugung Palace, among others.

\subsection{The active role of young designers}

It is crucial for young designers to play an active role in trading. Almost half of SFW's brands are under 5 years old and cannot afford to open their own stores. On a domestic scale, the increase of multi-shops in department stores, shopping malls etc., helps keep young designers in business. Whereas well-established brands have their own regular clientele, young designers expect to find new buyers and customers in the international market. Apart from supporting Fashion Week, Seoul's Metropolitan Government and the Ministry of Culture, Sports and Tourism, through the Korean Creative Content Agency (KOCCA), encourage this expansion by funding, for instance, Seoul's 10 Soul (2010 to present) and Concept Korea.

The exporting amount of Korea's fashion manufacturing industry (Market Share data) was the world's third in the 1980s (after Hong Kong's and Italy's), sliding to fifth in the early1990s; then in 1995 it plunged to ninth and its downturn still continues (Hong et al., 2010). In the 1980s Korea was exporting garments made by a low-cost workforce but as labour cost increased, many manufacturers moved to countries cheaper in labour, such as Indonesia or Vietnam. This is why Korea's market share figures have dropped. However, the Seoul Metropolitan Government Fashion Advisory Team (2009) points out that Korea is the place where the entire process of the fashion industry is connected, from planning, design and manufacturing to distribution. This distinguishing feature of 
the Korean fashion industry could be applied to promising young designers willing to enter the international market. This would enable Korea to go back to its prime.

\section{Weaknesses}

An expert survey by Seoul Institute (2014) about the problems of SFW shows that the constitution of an independent professional operational body (32\%), a budget increase (27\%), legislation on related business and system maintenance (27\%), etc. Kim (2012) points out the lack of an independent, sustainable operation system and organisation (the problems of hiring the agency), venues, differences in the brands' price ranges, the ineffectiveness of invitations to international buyers, the failure to present SFW as a business event, an identity problem and an equally confused concept of Fashion Week and its designers. Joo (2009) also points out the absence of international guests and buyers, lack of business results (little international trade, too much focus on celebrities attending), a chaotic operational system (SFAA, Seoul Collection, Seoul Fashion Centre and Seoul Fashion Association), lack of continuity in the support program for new and young designers, inadequate venue (SETEC, see 3.2. Venue), mismatch between business and publicity, absence of subsidiary events. Through background research focused on organisational issues, some of these problems can be reduced on the basis of some key factors.

\subsection{Organisational body}

After years of adjustment, Seoul Design Foundation finally became one organisational body in 2012. Far from being independent, however, SDF is part of the government. Further, it does not have enough staff members. In Seoul Metropolitan Government there are only four or five people in charge of fashion events. Their duties include not only Fashion Week but also, for instance, planning a fashion museum. So, it is hard to consistently focus on Fashion Week throughout the year ("Fashion industry team organisation chart", 2015). As to its running body, the BFC has 37 people working for Fashion Week and related events. In SDF, on the contrary, there are only 8 in charge of Seoul Fashion Week: these are the fashion team at the Fashion Culture head office run by the Seoul Metropolitan Government. Their duties include not only SFW but also the administrative role of the Export Support Centre, Fashion Creative Studio, the promotion of global fashion brands, the management of the Modelist contest (Seoul Design Foundation, 2015b). In this situation, hiring an agency is unavoidable. Table 7 shows the succession of SFW running bodies.

An agency normally consists of area specialists. Because SDF selects its agency after a fair and open bid, it can conduct a project efficiently and transparently. However, a new contract is made every season. This hinders long-term administration because the people in charge of one project change with every new contract. For the possibility of extending contracts, the agency focuses on short-term goals and immediately profitable events. Fashion Week, however, is a business tending not to produce instant results. Buying takes time and cannot be judged in the short term: if buyers want to make a contract with a young new brand, they normally look at one or two years of their activity for sustainability of brand identity, survival, etc. This kind of job is unsuitable for agencies, as they are mostly assessed by their immediate results. This prevents SFW from planning long-term, focused development strategies. After combining separate collections into one, there still remains to form one professional, independent organising body on the model of BFC. Further, including Caroline Rush, CEO of BFC, most of members are PR experts. This enables them to run strategic shortand long-term events. From March, the Seoul Design Foundation appointed a general manager, Kuho Chung (Vice-President of Fila Korea) in May 2015, in charge of the planning and management of Fashion Week from 2016 SS for two years (Lee, 2015). His is not a full-time position, though. In order to draw a whole, unified picture, planning should be accompanied by strong apolitical, nonprofit, organisation and staff.

\subsection{Guests}

Not only Joo's (2009) but also Han and Lee's research (2008) highlight the absence of buyers from Fashion Week through a survey of participants. The collection is open to the public. Fashion design students can access the show, which can be instructive; but it is a trade show, so the presence of visitors outside the industry

Table 7. SWF running bodies from 2005 to 2014

\begin{tabular}{|c|c|c|c|c|}
\hline Year & Funder & \multicolumn{2}{|c|}{ Runner } & Running show/ event \\
\hline $2005-2011$ & Seoul Metropolitan Government & Seoul Fashion Centre & $\begin{array}{l}\text { Nongovernmental } \\
\text { operational agency }\end{array}$ & \multirow{3}{*}{$\begin{array}{l}\text { running professional agency for } \\
\text { fashion show and convention }\end{array}$} \\
\hline $2012-2013$ & Seoul Metropolitan Government & \multicolumn{2}{|c|}{ Nongovernmental operational agency } & \\
\hline 2014 & Seoul Metropolitan Government & $\begin{array}{c}\text { Seoul Design Foundation } \\
\text { agency }\end{array}$ & $\begin{array}{l}\text { Non-governmental } \\
\text { operation agency }\end{array}$ & \\
\hline
\end{tabular}


can hinder the concentration on trade. The reason why the event should be open to public started from Korea's unusual distribution structure. Whilst in most Western countries buyers purchase items at fashion shows to be sold in department stores and independent boutiques, in Korea fashion brands operate quite independently and there is no notion of a 'buyer'. Without buyers, (and aside from the press) the seats are typically given or sold to students, which would be virtually unthinkable in other Fashion Weeks. In fact, fashion collections in Seoul have been regarded as a way of presenting designers' work rather than selling it. For this reason the credibility of SFW in the mind of the international fashion community has been rather shaky (Lee, 2013). Nowadays multi-brand fashion concept stores are increasing. The first multi store was 'G. Street 494' at Galleria department store in 1997, and started to increase from 2000. In 2007, there were 80 stores and $70 \%$ of them sold imported goods (Park, 2007). During 2015, the department store, shopping mall, road shop, online shopping mall, and even retail brands have been increasing its multi stores; department stores have changed their display into a zoning system, which was not specified by brands (Samsung Design Net, 2015). Inroads are being made into changing the system, but this is likely to take time.

If an internal problem is distribution, an external one is the combination of time and location: Seoul Fashion Week's schedule is late compared to other Fashion Weeks, and Korea is geographically distant from the main trade locations, that is, Europe and America (this will be discussed in 4.4. Time and Location). Due to the distance from the main trade locations, as much as $30 \%$ of the SFW budget is spent on guests' travel expenses, accommodation and stay. BFC too spends a significant amount of money on guests. However, it is always pointed out that there is an issue about the quality of both buyers and the press (Kim, 2012). This naturally means insufficient returns compared to monetary investment. Lee Kio points out some of SFW's problems in this area. One of them is that the number of contracts is low compared to other Fashion Weeks'. Myung You Seouk, a Hempel CEO, also stresses SFW's lower achievement compared to European, American and even Chinese fashion fairs (Lee, 2013).

For PR purposes, huge numbers of celebrities are invited to the show. However, because their attendance causes competition in media coverage and monopolises public focus, buyers and press can hardly concentrate on the show itself (Joo, 2009).

\subsection{Sponsorship}

SFW is wholly funded by Seoul's Metropolitan Government. Sole sponsorship can be a problem if it cuts down its investment. If an alternative cannot be found (it takes time to find sponsors), the event must be downsized. This obviously affects the credibility of Fashion Week. The ratio of dependency on government should be gradually decreased through funding from more diverse places and ways.

The sponsoring company supports products and services or spends money for its own promotion as suppliers and funders. So, the company logo and information should be visible. Although the sponsored party's homepage is the best permanent tool for exposure, there is no mention of sponsors on the SFW homepage.

\subsection{Time and location}

There is a fixed time for ordering next season's stocks for a department store or shop. Buyers set their budgets and items and draw up contracts during and just after the Fashion Week event, after watching the fashion shows. This is what normally happens in the four major Fashion Weeks. As table 6 shows, SFW takes place later than the Big 4, so most of the contracting between buyers and brands is over by then. It is always a problem to set the date, because of the impossibility to squeeze the Seoul event between the main Fashion Weeks, and this is due to the buyers' movements. If SFW is moved earlier than the Big 4, buyers can see it first, make their notes and postpone the contract to after they have watched the four Fashion Weeks. However, because of domestic sales and the manufacturing calendar, January or the beginning of February and August, or the beginning of September, are too early for designers to prepare their collections. The physical distance from the main markets is not the sole organisational problem; however, it can be considered in terms of SFW's targets. Its advantages include closeness to the Asian and Middle Eastern markets. If it is developed separately in the Asian market, schedule and venue matter much less. Because the mainstream market is Europe and the USA, trends start from there. Thus, it is helpful to have a stock list there to promote and sell to the Asian market. This issue should be developed further.

\subsection{Main event}

Over all, as can be seen in table 2, there is no consistency among the events scheduled. There used to be Fashion Take Off (2011 AW to 2012 AW), inspired by BFC's Fashion Forward program (Ham, 2011), as a bridge between Generation Next and Seoul Collection, to help young designers establish their brands. In 2012 AW the scale of Seoul Fashion Fair was a lot larger (200 brands in 2012 AW) than in 2015 SS (33), when it stopped. In 2014 SS there were many events scheduled, but in 2015 AW hardly any. Although it is a period of transition, it is better to keep up consistency. If one event is set, however, it is better to alter its contents through trial and error than cancel the whole thing. Continual change in the 
main event affects the credibility of SFW. Once the event is set, it is necessary to wait for a while to know its results. If SFW set up a long-term goal for the next 10-20 years, it can set a direction towards it. Seoul's current goal to be the world's 5th Fashion Week or fashion capital should be accompanied by more detailed and realistic directions.

If the first weakness is the problem of the persistency of the main event, the second one is the lack of different collections according to brand size. SFW only has Seoul Collection and Generation Next which both of which are under 5 years old. The differences between these two are show size and funding. There is no difference in their method. In the case of LFW, there are fashion show and presentation show. A presentation show features a small number of garments (normally more or less 20) on digital film, at a closer distance from the audience, with three or four shows in two hours. Guests can have a closer look at the clothes and talk business in-between shows, thus getting to know items and brands. Digital showcasing displays brand identity and images more widely. The showroom is actively run as a vibrant place for trade, not only for fashion show brands but also for accessories or small-sized brands.

\subsection{Supporting event}

Supporting events are for attracting guests or the public. They can be related with business, education, entertainment, etc. However, an official supporting event of SFW is Give Donation, which sells donated clothes from participating designers. In 2015 SS of LFW, there were numerous such events: Vodafone London Fashion Weekend, Live streaming of fashion show, BBC broadcasting of three documentary series, the highlights of fashion show broadcast in 19 major underground stations during the Fashion Week period, fashion film screenings, photobooth service (taking photos of guests, to print and share through Facebook), pop up store, eBay BFC contemporary shop, Oxford street, LFW special window display and style seminar at a main shopping street in London, Decoded Westfield (online opinion making competition), an exhibition of fashion photographer, David LaChapelle at the bus stop near Somerset House, the location of LFW and parties in collaboration with magazines or big clothing companies. In addition, there were LFW digital Talks about important issues in fashion, live-streamed worldwide. One of themes in 2015 SS was: "can you bring real luxury online?". This issue covers industry and academia: beside being commercial, it also takes into account the future of fashion. If SFW organised more supporting events for education and promotion, for professionals and non-professionals, for the domestic and international markets, it would be helpful for both the present and the future of SFW.

\subsection{Online promotion}

Homepage and social media are effective PR tools, as there news can be updated, responses can be seen almost in real time and communication can take place bilaterally. However, the SFW homepage and social media do not function well. The homepage is not fully transferring information about Fashion Week and its designers. They check the details of SFW and its designer information through its homepage and, nowadays, the social media. Many people use social media (Twitter, Facebook or Instagram) which can be updated on a daily bases. Further, it would be efficient to have links from designer profiles to their social media to learn the latest about their brands. Finally, updating news and fashion shows in real time and disseminating the information through the social media are effective promotional tools that can save money on PR. So, it is natural for Fashion Week to invite power bloggers such as buyers and press as guests.

Apart from updating images and news, LFW uses live streaming of fashion shows. This can overcome the time and location problem because the internet can be accessed anywhere, in real-time, and at any time. In 2015 SS, 196 countries watched live streams of LFW ("London Fashion Week", 2015). This would otherwise be impossible in the actual fashion show.

As to technical problems, it is important to collaborate with IT companies; in 2015 SS, Topshop collaborated with Facebook and Instagram, and Burberry with Twitter, with cloud-based real-time TV and video clips shared through Twitter for world-wide communication.

\subsection{The characteristics of Fashion Week}

LFW is renowned for showcasing creative young new designers; New York Fashion Week is for a practical and saleable collection. Then, what is the distinguishing feature of SFW? It needs to be specific in order to attract buyers and press to the event. It needs to reflect what is and what will be strong in Korean fashion and SFW. The vibrant presence of young designers can be one solution, so SFW can be settled as a showcase of young designers in Asia, similarly to LFW.

Advanced Korean IT technology can be used as an innovation hub in Fashion Week. LFW combines fashion showcase with digital tech by showing Fashion films, digital presentation shows, live streaming and real-time communication. For example, House of Holland collaborates with Metail, an online fitting room, by creating a customised model called MeModel according to the wearer's size and tastes. Customers can try the garments on in real time when watching the live-streamed catwalk show. If this technique is matched with young designers' collections, it will be enough to attract young audiences, with their buyers and press. 
Table 8. The strengths and weaknesses of SFW

\begin{tabular}{|c|c|c|}
\hline \multirow{3}{*}{ Strengths } & One organisation & Union finally achieved after a long struggle to combine since the launch \\
\hline & Venue & $\begin{array}{l}\text { Settled as a fashion market and industry hub in Seoul, centrally located and well-served by public } \\
\text { transport, with easy access to designers }\end{array}$ \\
\hline & $\begin{array}{l}\text { The active role of young } \\
\text { designers }\end{array}$ & Almost half of designers in SFW have been in business for under five years \\
\hline \multirow{8}{*}{ Weaknesses } & Organisational body & $\begin{array}{l}\text { Independent organisational body with PR professional staff (strong apolitical, non-profit organisation } \\
\text { and staff ) in charge of Eashion Week all year round is needed }\end{array}$ \\
\hline & Guests & $\begin{array}{l}\text { Lack of buyers and press from the unusual distribution structure Insufficient returns compared to } \\
\text { monetary investment } \\
\text { Huge numbers of celebrities: hard to focus on trade }\end{array}$ \\
\hline & Sponsorship & sole governmental sponsorship \\
\hline & Time and location & late Fashion Week calendar compared to major Fashion Weeks, distance from main market \\
\hline & Main events & inconsistency, unclear distinction between Seoul Collection and Generation Next \\
\hline & Supporting events & not enough events to attract guests and to supplement main events \\
\hline & Online promotion & lack of information, no online promotion \\
\hline & The characteristics & no distinguishing feature to attract buyers and press \\
\hline
\end{tabular}

\section{Conclusion}

Seoul Fashion Week, a biannual fashion showcase, has put significant time, effort and money into becoming one of the world's leading Fashion Weeks. The strengths and weaknesses of SFW have been analysed on the basis of a number of headings (organisational body, designers, visitors, schedule, venue, events) drawn from the homepage of London Fashion Week, its most successful counterpart. Throughout its 16-year old history, SFW has grown to the size of a world-renowned Fashion Week. Its greatest achievement is that it finally has combined a number of different collections into one. Secondly, the venue seems now settled as DDP, in the design and fashion culture centre of Seoul. Finally, a number of young designers are actively participating and making new contracts. However, there have been a number of problems ahead of becoming the world fifth Fashion Week. 1), despite having one organisational body, SFW is not independent and there are not enough specialised staff: the event is actually run by private operation agencies; 2), it should be focused on trade rather than on the general public; 3), it depends exclusively on government funding; 4), the SFW schedule is incompatible with those of the major Fashion Weeks and its location is far from the American and European fashion markets; 5), the main events are not sustainable; 6 ), there are not enough supporting events to attract guests and to supplement main events; 7), the SFW homepage and social media are not actively used for promotion; finally, there is no distinguishing feature to SFW yet. Therefore, if fast growth and the active role of young designers mean that its future is positive, SFW should have one independent, active, powerful, specialised organisation in order to conduct sustainable, long-term planning, reduce governmental dependency and recruit private funding.

An awareness of SFW's current situation and problems means that more down-to-earth solutions can be found. Fashion is a high added-value, yet very sensitive, industry. If SFW overcomes its weaknesses and learns from successful Fashion Weeks, it will become the world's fifth event of this kind, which is the aim of the Seoul Metropolitan Government. If the following research is accompanied by a development plan, it would be possible to achieve this goal; it can be useful to conduct studies of successful Asian cases, such as China Fashion Week, as well as planning the future direction of SFW through other, smaller, Fashion Weeks. An in- depth interview with designers would also help towards a fuller diagnosis of the situation. Invitations of international buyers and press from Europe and America should be considered with more attention. This study only deals with organisation. However, there are many other issues, such as designers and their prices or products range for trade. If this research is followed up, it could help towards setting up a realistic plan for an appropriate governmental policy.

\section{References}

Council of Fashion Designers of Korea. (2015). About CFDK. Retrieved August 3, 2015, from http://www.cfdk.org/about/history.asp

Dongdaemoon Design Plaza (DDP). (2015). About DDP. Retrieved August 3, 2015, from http://www.ddp.or.kr/DI010007/getInitPage. do?MENULEVEL $=1 \quad 1 \quad 1$

'Fashion industry team organisation chart'. (2015). Seoul Metropolitan Government. Retrieved September 23, 2015, from http://namesearchapp.seoul.go.kr/main/org/org_chart_bon.jsp?dept_cd=33150000

Ham, M. J. (2011, May 30). 2011 2012 F/W 서울패션위크 신진 디 자이너들 9 팀의 무대 '패션테이크오프' 열기 후끈 [The 
Excitement of 2011 2012 F/W Seoul Fashion Week 9 Young Designers' Stage, Fashion Take Off]. Fashionbiz. Retrieved August 16, 2015, from http://www.fashionbiz.co.kr/TC/main.asp?cate= $2 \& \mathrm{idx}=122578$

Han, J. Y., \& Lee, S. J. (2008). An exploratory study for the market of Seoul collection - From the collection participants' perspective -. Journal of the Korean Society of Clothing and Textiles, 32(4), 562-572. doi:10.5850/JKSCT.2008.32.4.562

Hong, K. H., Lee, J. S., Kim, Y. M., Yang, J. O., \& Lee, Y. J. (2010). The production situations of apparel sewing company and the perceptions about the mass customization. Fashion \& Textile Research Journal, 12(2), 162-171.

Joo, B. R. (2009). Research on "Korean" collection as high added value fashion industry - By comparison of status and characteristics of domestic and international collections. Journal of the Korean Society of Design Culture, 15(4), 520-531.

Kim, H. E. (2015). A study on the characteristics and trends of sustainable fashion through Esthetica at London Fashion Week. Fashion \& Textile Research Journal, 17(2), 168-177. doi:10.5805/ SFTI.2015.17.2.168

Kim, H. J. (2012). Analysis on the current status of Seoul Fashion Week and study on the methods of its mid- and long-term development. Journal of Korean Society of Design Science, 25(3), 298-314.

Kim, H. M. (2015, April 28). 서울패션 위크 이대로 괜 챻은가 [Is it okay Seoul Fashion Week as it is?]. bntnews. Retrieved August 15, 2015, from http://bntnews.hankyung.com/apps/news?popup= $0 \&$ nid $=08 \& \mathrm{c} 1=08 \& \mathrm{c} 2=08 \& \mathrm{c} 3=00 \&$ nkey $=201504101211473 \& \mathrm{~m}$ ode $=$ sub_view

Kim, S. M. (2008a). A study on the development strategies of SFAA, Seoul Collection. Unpublished master's thesis, Dongduk Womens University, Seoul.

Kim, Y. I. (2008b, October). 서울 패션산업의 글로벌 경쟁력을 위한 발 전 방향 [The development strategies of the Seoul Fashion Industry]. 서울경제 [Seoul economics], pp. 3-12.

Lee, C. Y. (2013, April 18). 서울패션위크에 바란다 - 전문가 설 문조사 [Wishes to Seoul Fashion Week - Expert Survey]. Apparel News. Retrieved April 11, 2015, from http://www.apparelnews.co.kr $/ 21 \mathrm{cNet} /$ print.php?uid $=46221 \& \mathrm{sbj}=\ldots 5 \% \mathrm{~B} 4 \% \mathrm{D} 9 \% 20-$ $\% 20 \% \mathrm{C} 0 \% \mathrm{FC} \% \mathrm{~B} 9 \% \mathrm{AE} \% \mathrm{~B} 0 \% \mathrm{~A} 1 \% 20 \% \mathrm{BC} \% \mathrm{~B} 3 \% \mathrm{~B} 9 \% \mathrm{AE} \% \mathrm{C} 1$ $\% \mathrm{~B} 6 \% \mathrm{BB} \% \mathrm{E} 7$

Lee, C. Y. (2015, May 14). 정구호, 서울패션위크 총감독으로 돌 아온다 [Chung Kuho is coming back as a general manager of Seoul Fashion Week]. Apparel News. Retrieved August 12, 2015, from http://www.apparelnews.co.kr/naver/view.php?iid=55096

Lee, K. O. (2014, October 21). 서울패션위크 세계적 축제로 발 돋움해야 [In search of Seoul Fashion Week to be a Global festival]. Sports Khan. Retrieved April 10, 2015 from http:// sports.khan.co.kr/news/sk_index.html?cat=view\&art_id= $201410212350103 \&$ sec $\_$id $=563101 \& p t=n v$

'London Fashion Week \& British Fashion Industry Facts \& Figures AW 15'. (2015). Retrieved September 27, 2015, from http:// www.londonfashionweek.co.uk/news_detail.aspx?ID=705

Mercedes-Benz Fashion Week Homepage. (2015). About MercedesBenz Fashion Week. Retrieved September 9, 2015, from http:// mbfashionweek.com/about

Noh, N. (2007). 노라 노, 열정을 디자인하다 [Nora Noh, designing ambition]. Seoul: Hwangkmnachimpan.

Park, S. H. (2000). Comparative study on fashion trends between Korean and foreign fashion collections in the 1990s. Unpublished master's thesis, Seoul National University, Seoul.

Park, S. H. (2007). 패션 유통 핵심 축으로 부상 [Select Shop becomes the core of fashion retail]. Apparel News. Retrieved September 4, 2015, from http://www.appnews.co.kr/main/pnews.php?table= paper_news\&query $=$ view\&uid $=42629 \& p=$

Samsung Design Net. (2015, May 21). 2015 S/S 유통 동향[2015 S/ S Market/Retail Report]. Retrieved August 10, 2015 from http:// www.samsungdesign.net/Market/MarketReport/content.asp?an $=40116 \& \mathrm{glChk}=\mathrm{G} \&$ block $=0 \&$ page $=1 \& \mathrm{cnt}=9$

Seoul Design Foundation. (2015a). 2015년 패션 봉제 사업 종합계획 [A fashion sewing comprehensive plan in 2015]. Retrieved April 4, 2015, from http://opengov.seoul.go.kr/sanction/4120153?from = policy

Seoul Design Foundation. (2015b). 패션팀 조직도 [Fashion team organization chart]. Retrieved April 24, 2015, from http:// www.seouldesign.or.kr/info2/group04.jsp

Seoul Institute. (2014). 서울시 패션정책 마스터 플랜 수립을 위한 연구 [The research into establishing master plan of Seoul Metropolitan Governmental Fashion Policy]. Seoul: The Seoul Institute.

Seoul Metropolitan Government Fashion Advisory Team. (2009). 2009서울시 패션산업 발전 방안 [A Fashion Industry Development scheme of Seoul Metropolitan City 2009]. Seoul: Seoul Fashion Centre.

(Received 7 October 2015; 1st Revised 11 November 2015; 2nd Revised 17 November 2015; 3rd Revised 19 November 2015; Accepted 27 November 2015)

(C) 2015 (by) the authors. This article is an open access article distributed under the terms and conditions of the Creative Commons Attribution license (http://creativecommons.org/licenses/by/3.0/), which permits unrestricted use, distribution, and reproduction in any medium, provided the original work is properly cited. 